\title{
MODELAMIENTO Y SIMULACIÓN DE UN SISTEMA NO CONVENCIONAL DE LEVANTAMIENTO MULTIFASICO
}

\author{
Julio C. Pérez Angulo1*, Manuel E. Cabarcas Simancas², Nicolás Santos Santos ${ }^{3}$
}

*A quien debe dirigirse la correspondencia

\section{RESUMEN}

La importancia del flujo multifásico se origina en que cuando el fluido producido se desplaza desde el yacimiento hacia la superficie, se libera energía tanto en el flujo vertical como en el flujo horizontal; esta energía o presión está inmersa en el fluido mientras está en el yacimiento. Por lo tanto, es necesario realizar un buen diseño de los equipos del pozo, líneas de flujo, estranguladores, separadores, y demás conexiones. Para un diseño óptimo, es necesario realizar un estudio detallado del comportamiento del flujo multifásico en cada componente. El presente artículo presenta el desarrollo de un Software que permite determinar mediante correlaciones y modelos mecanísticos los perfiles de presión y temperatura que se presentan a lo largo de las tuberías. El Software está desarrollado en una interfaz gráfica en lenguaje de programación Java y fue validado en el Software comercial PIPESIM. De esta manera se podrá estudiar más a fondo el comportamiento del flujo multifásico en tubería vertical y horizontal.

Palabras clave: Hold Up, Patrones de flujo, flujo Multifásico.

\section{MODELING AND SIMULATION OF AN UNCONVENTIONAL LIFTING MULTIPHASE SYSTEM}

\begin{abstract}
ABSTRAC
The importance of the multiphase flow is caused in that when the produced fluid is displaced from the reservoir to the surface, energy is released in both the vertical and horizontal flow; this energy or pressure is possessed by the fluid while it is in the reservoir. Therefore, it is required to make a good design teams well, flowlines, throttles, separators and other connections. For optimum design, it is necessary to conduct a detailed study of the behavior of multiphase flow in each component. This paper presents the software developed which allows determine through correlations and mechanistic models, the profiles of pressure and temperature that occur along the pipes. The software was developed in a graphic interface in Java programming language and was validated with PIPESIM commercial software. In this way it can be further studied the behavior of multiphase flow in vertical and horizontal pipe.
\end{abstract}

Keywords: Hold Up, Flow Patterns, Multiphase flow.

1. Ingeniero de petróleos. Centro de Investigación del Gas y del Petróleo. Universidad Industrial de Santander, UIS. Bucaramanga. Colombia. E-mail: jperez@uis.edu.co

2. Magister en Ingeniería Química. Centro de Investigación del Gas y del Petróleo. Universidad Industrial de Santander, UIS. Bucaramanga. Colombia. E-mail: cabarcas@uis.edu.co

3. Magister en Ingeniería de Hidrocarburos. Grupo de Modelamiento de Procesos de Hidrocarburos. Universidad Industrial de Santander, UIS. Bucaramanga. Colombia. E-mail: nicolas@uis.edu.co 


\section{INTRODUCCIÓN}

En la industria petrolera es de gran importancia determinar el comportamiento del flujo multifásico en tubería, pues cuando el fluido producido se desplaza desde el yacimiento hacia la superficie, se libera energía en el flujo vertical y horizontal, esta energía o presión es poseída por el fluido mientras este se encuentra en el yacimiento; y es en este flujo donde se producen las mayores pérdidas de energía, por esto es necesario predecir de manera óptima los gradientes de presión y de temperatura, conociendo extensivamente el comportamiento detallado de los fluidos dentro de la tubería, es posible manipular las condiciones de trabajo para aprovechar al máximo la presión de flujo, la cantidad de gas, y/o el patrón de flujo presente, para economizar en equipos de transporte, red de tuberías y accesorios, medidores, equipos de separación y otros, de acuerdo con las características del sistema y la topografía de la región considerada; para este fin se realizan pruebas de presión y/o se emplean simuladores, algunos son comerciales pero los altos costos limitan el acceso a estos. Como solución, se consideró de gran ayuda para la academia desarrollar un simulador de fácil acceso y de interfaz amigable con el usuario, con capacidad para calcular los perfiles de presión y temperatura bajo condiciones de flujo multifásico. En el desarrollo de este artículo, se presenta en primer lugar la metodología aplicada en el desarrollo del software, es decir, la descripción de los modelos seleccionados para la programación, que permiten modelar el comportamiento del flujo y predecir el perfil de presión y temperatura, en tubería vertical y horizontal. Posteriormente se encuentran los resultados obtenidos después de la programación del software, además de los obtenidos por el software comercial PIPESIM para cada modelo utilizado, con el fin de realizar la comparación entre estos. Finalmente, se presentan las conclusiones obtenidas en el desarrollo de este artículo, las cuales permitirán determinar el cumplimiento de los objetivos planteados.

\section{MÉTODO}

Inicialmente se realizó una búsqueda bibliográfica para identificar las correlaciones empíricas y modelos mecanísticos desarrollados por diferentes autores para cálculos de presión y temperatura a lo largo de tubería vertical y horizontal. Los más empleados en la industria fueron validados en Excel y los resultados comparados con el software PIPESIM tomando como valores reales los de PIPESIM, los que obtuvieron los menores errores porcentuales fueron seleccionados para la programación en Java, por consiguiente el software resultado de esta investigación cuenta con 3 correlaciones empíricas y un modelo mecanístico para cada tipo de tubería.

\section{MARCO TEÓRICO}

\section{CORRELACIONES EMPÍRICAS PARA PERDIDAS DE PRESION EN TUBERIA}

Diversos investigadores han desarrollado correlaciones de flujo multifásico en tuberías verticales y horizontales, basándose en los principios termodinámicos y de flujo de fluidos, pero principalmente en observaciones empíricas limitadas por caídas de presión por fricción, diámetros de tuberías, características de los fluidos utilizados, geometría y condiciones de flujo, y relaciones gas líquido.

Varias correlaciones para predecir las pérdidas de presión en tubería vertical han sido publicadas. Debido a la complejidad del flujo multifásico, las correlaciones propuestas por necesidad son altamente empíricas. [1]

La ecuación de gradiente de presión general se define así (1):

$$
\frac{d p}{d z}=\left(\frac{d p}{d z}\right) e \boldsymbol{l}+\left(\frac{d p}{d z}\right) f+\left(\frac{d p}{d z}\right) \boldsymbol{a c c}
$$

\subsection{TUBERÍA VERTICAL}

\subsubsection{Correlación de Beggs \& Brill}

El método de Beggs \& Brill es basado en trabajos hechos con mezclas de agua y aire para diferentes condiciones. Esta correlación, se enfatiza en calcular las propiedades que afectan las caídas de presión de flujo multifásico a diferentes grados de inclinación de la tubería. Para este método la ecuación de caída de presión para la fase de gas, líquida o ambas se expresa de la siguiente manera (2).

$$
-\frac{d p}{d z}=\frac{\frac{g}{g c} \operatorname{sen} \theta(\rho l f l+\rho g(1-f l))+\frac{f m G m v m}{2 g c d}}{1-\frac{(\rho l f l+\rho g(1-f l)) v_{m} v_{g}}{p g_{c}}}
$$

Esta ecuación se puede reducir a una sola fase líquida si $f 1$ tiende a uno o una fase gaseosa si $f 1$ tiende a cero. También, puede ser aplicada a tuberías verticales u horizontales si a ángulo es igual a $90^{\circ} \mathrm{o} 0^{\circ}$ grados. 
La Tabla 1, muestra los rangos entre los cuales se encuentran las limitaciones del método. [2]

Tabla 1. Listado de Limitaciones.

\begin{tabular}{ccc} 
Variable & Rango & Unidades \\
\hline Caudal de Gas & $0-300$ & Mpies3/dia \\
\hline Caudal de Liquido & $0-30$ & $\mathrm{Gal} / \mathrm{min}$ \\
\hline Presión promedio del sistema & $35-95$ & $\mathrm{psia}$ \\
\hline Diámetro de la tubería & $1-1,5$ & $\mathrm{pg}$ \\
\hline Colgamiento del liquido & $0-0,870$ & $\mathrm{~N} . \mathrm{A}$ \\
\hline Gradiente de Presión & $0-0,8$ & Psi/pie \\
\hline
\end{tabular}

\subsubsection{CORRELACIÓN EMPÍRICA PoettMan \& Carpenter}

Poettman \& Carpenter desarrollaron un método semiempírico utilizando diámetros de tubería de producción de 2, 2, 3 pulgadas en el que incorporaron la ecuación general de energía y datos de 34 pozos fluyentes de aceite y 15 con bombeo neumático. El aceite, gas y agua fueron considerados para estar en una sola fase, y no se trató de determinar la correlación de colgamiento. Toda la pérdida de energía incluyendo el resbalamiento y líquidos de emergencia se introdujeron en un factor de pérdida de energía el cual tomó como constante en toda la longitud de la tubería. También se asumió el flujo vertical de aceite, gas y agua como turbulento, además de no tomar en cuenta los patrones de flujo. La ecuación general para el cálculo de la presión es (3).[2]

$$
\left(\frac{d p}{d z}\right) T=\frac{1}{144}\left[\bar{\rho} m+\frac{f W m^{2}}{7,413 * 10^{10} \bar{\rho} m\left(\frac{d}{12}\right)^{5}}\right.
$$

La correlación de Poettman \& Carpenter sique siendo usada extensamente en los campos bajo las siguientes condiciones (Tabla 2):

Tabla 2. Condiciones de uso para correlación Poettman \&Carpenter

\begin{tabular}{ccc}
\hline Variable & Rango & Unidades \\
\hline Diámetro & $2-2(1 / 2)$ y 3 & pg. \\
\hline Relación Gas líquido & $<1500$ & Pies3/bbl \\
\hline Caudales & $>400$ & Bbl/día \\
\hline Viscosidad & 5 & $\mathrm{cp}$. \\
\hline
\end{tabular}

\subsection{TUBERÍA HORIZONTAL}

\subsubsection{CoRrelación EMPÍRICA DE EATON ET AL. ( 1967)}

La correlación propuesta por estos autores fue desarrollada a partir de data proveniente de un sistema de tubería horizontal de 2 y 4 pulgadas de diámetro y aproximadamente 1700 pies de longitud. Eaton y compañía proponen correlaciones para determinar el factor de fricción y la fracción de líquido con deslizamiento. La ecuación general para el cálculo de la presión es (Ecuación 4):

$$
\begin{aligned}
\Delta L=\frac{2 g_{c} d}{12 w_{m} V_{m}{ }^{2} f} & {\left[144 \Delta P=\left(\frac{w_{L}}{\rho_{L}}+\frac{w_{G}}{\rho_{G}}\right)\right.} \\
& \left.-\frac{w_{L} \Delta V_{L}{ }^{2}+w_{G} \Delta V_{G}{ }^{2}}{2 g_{c}}\right]
\end{aligned}
$$

En la Tabla 3, se muestran los parámetros de correlación de Eaton et al.

Tabla 3. Condiciones de uso para correlación Eaton et al.

\begin{tabular}{|ccc|}
\hline Variable & Rango & Unidades \\
\hline Flujo de Gas & $0-10$ & $\mathrm{MMSCF} / \mathrm{d}$ \\
\hline Flujo de líquido & $50-5500$ & $\mathrm{Bbl} / \mathrm{d}$ \\
\hline Presión promedio del sistema & $70-950$ & $\mathrm{psig}$ \\
\hline Viscosidad del liquido & $1-13,5$ & $\mathrm{cp}$ \\
\hline Hold Up de líquido & $0-1$ & N.A \\
\hline
\end{tabular}

\subsubsection{CORRELACIÓN EMPÍRICA DE BEgGs \& BRILL (1973)}

La correlación de Beggs and Brill (1973) fue desarrollada en 584 pruebas tomadas de datos obtenidos experimentalmente de pruebas de arreglos a pequeña escala. La prueba consistió en una tubería de acrílico de 1 y 1,5 pulgadas de diámetro y 90 pies de longitud, la cual tenía un mecanismo que lograba inclinar la tubería de horizontal a vertical y los fluidos utilizados en aire y agua. La ecuación general para el cálculo de la presión es (Ecuación 5):

$$
\left(\frac{d p}{d L}\right)_{t}=\frac{\frac{f G_{m} V_{m}}{2 g_{c} d 12}}{1-\frac{\rho_{m} V_{m} V_{s g}}{g_{c}(p+14,7)(144)}}
$$


En la Tabla 4, se pueden apreciar los parámetros de correlación de Beggs \& Brill (1973).

Tabla 4. Condiciones de uso para correlación Beggs \& Brill (1973)

\begin{tabular}{|ccc|}
\hline Variable & Rango & Unidades \\
\hline Caudal de Gas & $0-300$ & MMSCF/dia \\
\hline Caudal de Liquido & $0-30$ & Gal/min \\
\hline Presión promedio del sistema & $35-95$ & psia \\
\hline Gradiente de Presión & $0-0,8$ & Psi $/ \mathrm{ft}$ \\
\hline Hold Up de liquido & $0-0,870$ & N.A \\
\hline
\end{tabular}

\section{PERDIDAS DE CALOR EN TUBERÍA}

Por definición, el calor es la energía que se transfiere como resultado de una diferencia o gradiente de temperatura. Matemáticamente es una cantidad vectorial, en el sentido que fluye de regiones de altas temperaturas a regiones de bajas temperaturas.

Los mecanismos de transferencia de calor se pueden clasificar de acuerdo con la relación existente entre las formas de energía y las sustancias de trabajo. Estos mecanismos son: conducción, convección y radiación [3].

\subsection{PÉRDIDAS DE CALOR EN TUBERÍA VERTICAL EN POZO}

\subsubsection{Calculo del coeficiente de TRANSFERENCIA DE CALOR POR RADIACIÓN}

El coeficiente de transferencia de calor por radiación, $h r$ es obtenido a partir de la ecuación (6)

$$
h_{r}=\frac{\sigma\left(T_{i n s}^{* 2}+T_{c i}^{* 2}\right) *\left(T_{i n s}^{*}+T_{c i}^{*}\right)}{\frac{1}{\varepsilon_{i n s}}+\frac{r_{i n s}}{r_{C i}}\left(\frac{1}{\varepsilon_{i n s}}-1\right)}
$$

Donde el asterisco denota temperaturas absolutas, $\sigma$ representa la constante de Stefan-Boltzmann con un valor de $1,713 \times 10^{-9} / \mathrm{ft}^{2} . \mathrm{hr}^{\circ} \mathrm{R}$, Eins y $\varepsilon_{c i}$ son las emisividades de la superficie externa de la capa de aislamiento y de la superficie interna del tubing. Es difícil obtener valores exactos de las emisividades, los cuales dependen del acabado de la superficie y del factor de vista, entre otras variables. Adicionalmente, se requiere de un procedimiento iterativo debido a que la temperatura al interior del casing y la temperatura de la superficie externa del aislamiento se necesitan. En la mayoría de los casos, la diferencia de temperatura a través del anular, y por lo tanto $h_{r}$, es usualmente muy pequeña.

\subsubsection{Calculo del coeficiente} DE TRANSFERENCIA DE CALOR POR

\section{CONVECCIÓN EN EL ANULAR}

Cuando un fluido a una temperatura dada está en contacto con una superficie a diferente temperatura, la transferencia de calor tiene lugar no solamente debido a la conducción, sino también debido al fenómeno conocido como convección natural. La convección natural se produce porque la densidad del fluido depende de la temperatura. La diferencia de temperatura, y por lo tanto la diferencia de densidad entre la masa de fluido y el fluido cercano a la superficie causa la circulación de fluido, la cual a su vez, mejora la transferencia de calor (Ecuación 7).[4]

$h_{c}=0,1\left(\frac{d_{c i}}{d_{t o}}\right)^{0,15}\left(\frac{\rho_{a}^{2} \beta g\left(T_{i n s}-T_{c i}\right)}{\mu_{a}{ }^{2}}\right)^{0,3} K_{a} d_{t o}{ }^{-0,1}(\operatorname{Pr})^{0}$

2.1.3 Calcular el coeficiente global dE TRANSFERENCIA DE CALOR (ECUACIÓN 8)

$$
\frac{1}{U_{t o}}=\frac{r_{t o} \operatorname{In}\left(\frac{r_{i n s}}{r_{t o}}\right)}{K_{i n s}}+\frac{1}{\left(h_{c}+h_{r}\right)}+\frac{r_{t o} \operatorname{In}\left(\frac{r_{w b}}{r_{c o}}\right)}{K_{c e m}}
$$

\subsubsection{Calcular LAS PÉRDidas} DE CALOR (ECUACIÓN 9)

$$
Q=-2 \pi r_{t c} U_{t o}\left(T_{f}-T_{w b}\right)
$$

\subsubsection{Calcular EL GRAdiENTE DE} TEMPERATURA (ECUACIÓN 10, 11, 12,13) [4]

$$
\begin{gathered}
W_{L}=\frac{5,6146 * q_{l} * \bar{\delta}_{l}}{86400} * 3600 \\
W_{g}=\frac{0,0764 * \Upsilon_{g} * q_{g}}{86400} * 3600 \\
W_{m}=W_{L+} W_{g} \\
\frac{d H}{d L}=\frac{q}{W_{m}}-\frac{g \operatorname{sen} \alpha}{J * g_{c}}
\end{gathered}
$$

\subsection{PÉRDIDAS DE CALOR EN TUBERÍA HORIZONTAL EN SUPERFICIE}

El flujo en tubería horizontal considerado en el desarrollo del software producto del presente artículo, fue el de las líneas de superficie. En estas líneas intervienen siempre los tres mecanismos de transferencia de calor. En 
general estas pérdidas no son fáciles de calcular, pues dependen de las condiciones del medio ambiente, las cuales cambian constantemente.

2.2.1 Calculo de las propiedades del AIRE (ECuAciones 14, 15, 16, 17,18):

$$
\begin{aligned}
& K_{a}=0,01328+2,471 * 10^{-5} T-4,247 \\
& * 10^{-9} \mathrm{~T}^{2} \\
& C_{p a}=0,2382+1,390 * 10^{-5} T+1,027 \\
& * 10^{-8} \mathrm{~T}^{2} \\
& \rho_{a}=0,0771-8,848 * 10^{-5} \mathrm{~T}-3,744 \\
& * 10^{-8} T^{2} \\
& B_{a}=0,0024-0,757 * 10^{-5} T+0,169 * \\
& 10^{-7} \mathrm{~T}^{2}-0,148 * 10^{-10} \mathrm{~T}^{3} \\
& \mu_{a}=0,0400+6,155 * 10^{-5} T-1,220 * \\
& 10^{-8} \mathrm{~T}^{2}
\end{aligned}
$$

\subsubsection{Calculo del coeficiente de} CONVECCIÓN NATURAL $\left(h_{c a}\right.$ Ecuaciones $\left.19,20,21\right)$

- Velocidad del viento(V): 0 millas/hora:

$$
h_{c a}=0,53 \frac{k_{a}}{D_{i o}}\left[D_{i o}^{3}\left(T_{e}-T_{e n v}\right) \frac{g B a}{\left(V_{c a}^{2}\right)} \frac{C_{p a \mu a}}{(k a)}\right]^{0,25}
$$

- $\quad$ Para $1,0<8,8 D_{e a} V<50,0$

$$
h_{c a}=19,3 C_{p a} \frac{\left(\rho_{a} V\right)^{0,6}}{D_{i o}{ }^{0,4}}
$$

- $\quad$ Para $8,8 D_{e a} V<50,0$

$$
h_{c a}=8,0239 \frac{k_{a}}{D_{i o}}\left[\frac{5,28 \rho_{a} V D_{i o}}{\mu_{a}}\right]^{0,805}
$$

\subsubsection{Se CALCULA DE CoEficiente DE RADIACIÓN $\left(\boldsymbol{h}_{r}\right)$ :}

$$
h_{r}=0,1714 \times 10^{-8} F_{e}\left(T_{i o}^{2}+T_{e n v}^{2}\right)\left(T_{i o}+T_{e n v}\right)
$$

Donde:

$F_{e}=$ Factor de emisividad

\subsubsection{Se CALCULA EL COEFICIENTE}

GLOBAL DE TRANSFERENCIA DE CALOR

Y LA TASA DE PÉRDIDAS DE CALOR:

El calor perdido por el fluido caliente por unidad de tiempo y de longitud, considerando que la tubería posee un aislante será:

$$
\begin{gathered}
q=2 \pi r_{i o} U\left(T_{F}+T_{e n v}\right) \\
U=\left[\frac{r_{p i} \ln \left(r_{p o} / r_{p i}\right)}{K_{p}}+\frac{r_{p i} \ln \left(r_{i o} / r_{p o}\right)}{K_{i}}\right. \\
\left.+\frac{r_{p i}}{r_{i o}\left(h_{c a}+h_{r}\right)}\right]^{-1}
\end{gathered}
$$

El calor perdido por el fluido caliente por unidad de tiempo y de longitud, considerando que la tubería NO posee un aislante será:

$$
\begin{gathered}
q=2 \pi r_{p o} U\left(T_{F}-T_{e n v}\right) \\
U=\left[\frac{r_{p o} \ln \left(r_{p o} / r_{p i}\right)}{K_{p}}+\frac{1}{\left(h_{c a}+h_{r}\right)}\right]^{-1}
\end{gathered}
$$

La aplicación de la conservación de energía para flujo de fluido en tuberías, requiere que un segmento dado de la tubería la energía entrante, menos la energía saliendo, más la energía transferida para o del medio circundante debe ser igual a la tasa de la energía acumulada:

$$
\frac{d H}{d L}+\frac{g \operatorname{sen} \alpha}{J g_{c}}+\frac{V_{m}}{J g_{c}} * \frac{d V_{m}}{d L}=\frac{q}{W_{m}}
$$

Despejando el diferencial de entalpia:

$$
\frac{d H}{d L}=\frac{q}{W_{m}}-\frac{V_{m}}{J g_{c}} * \frac{d V_{m}}{d L}-\frac{g \operatorname{sen} \alpha}{J g_{c}}
$$

Es decir:

$$
\left(\frac{d H}{d L}\right) t=\left(\frac{d H}{d L}\right) h t+\left(\frac{d H}{d L}\right) e+\left(\frac{d H}{d L}\right) a
$$

Igual que con el gradiente de presión, el gradiente de la entalpia tiene tres componentes: transferencia de calor, tiene fuerte dependencia en la entalpia total; aceleración, que es despreciable y elevación, del producto de la gravedad que es despreciable. 


\subsubsection{Se calcula la Entalpia total}

Para flujo en tubería horizontal se tiene:

$$
\begin{gathered}
\left(\frac{d H}{d L}\right) t=\left(\frac{d H}{d L}\right) h t \\
\left(\frac{d H}{d L}\right) t=\frac{q}{W_{m}}
\end{gathered}
$$

Además:

$$
\left(\frac{d H}{d L}\right) t=C_{e} \frac{d T}{d L}
$$

Donde:

$C_{e}=$ Calor específico del fluido, $B T U / l b^{\circ} \mathrm{F}$

$\frac{d T}{d L}=$ Gradiente de temperatura, ${ }^{\circ} F / f t$

\subsubsection{SE CALCULA EL GRADIENTE DE} TEMPERATURA

$$
\frac{d T}{d L}=\frac{\left(\frac{d H}{d L}\right)_{t}}{C_{e}}
$$

Así, a partir del gradiente de temperatura, se determina la temperatura del fluido en cada punto de la tubería, con lo que se construye el perfil de temperatura (Temperatura vs. Distancia) para flujo en tubería horizontal en superficie.

\section{MODELO MECANÍSTICO PARA PÉRDIDAS DE PRESIÓN EN TUBERÍAS}

Un modelo mecanístico es un modelo matemático que explica el comportamiento de flujo multifásico en tubería incorporando todas las variables que están involucradas en este fenómeno. Complementando la parte teórica con datos de campo o de laboratorio apropiados, esta aproximación produce métodos de predicción que representan un adelanto significativo sobre las correlaciones empíricas generalizadas.

Una de las características de este método es la existencia de diferentes patrones de flujo en los sistemas multifásicos. El primer objetivo es determinar el patrón de flujo de un sistema dado. Luego, para cada patrón de flujo se desarrolla un modelo matemático apropiado, el cual predice la hidrodinámica del flujo y la transferencia de calor para este.
El flujo multifásico está caracterizado por un gran número de variable de flujo, casi el doble que para flujo de una sola fase. Su configuración también es muy compleja. Esto es especialmente cierto para el sistema gas-líquido con una fase compresible y una fase deformable, lo cual hace a las correlaciones empíricas imprácticas [5].

\subsection{MODELO MECANÍSTICO DE ANSARI ET AL. TUBERÍA VERTICAL}

Este modelo es un análisis exhaustivo del flujo. Está compuesto por un modelo de predicción de patrón de flujo y un grupo de modelos mecanicistas independientes los cuales predicen características como el Hold Up y la caída de presión en flujo burbuja, tapón y anular. A continuación se muestra el mapa de los patrones estudiados [6].

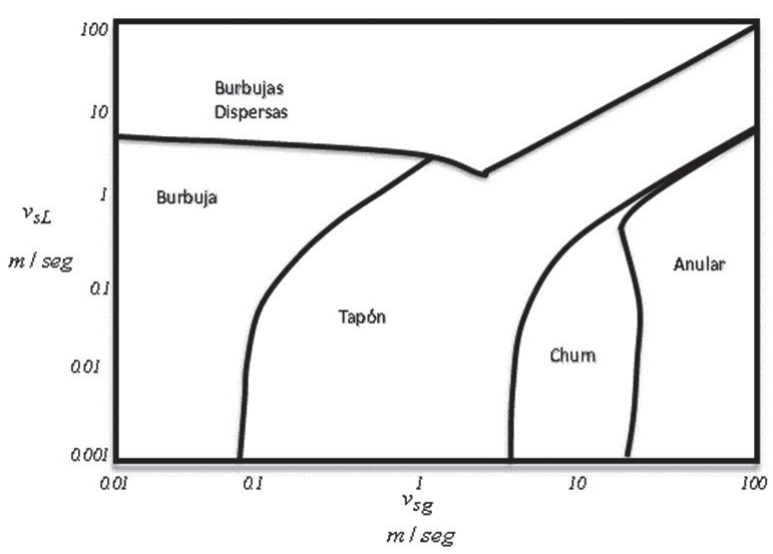

Figura 1. Mapa de patrones de flujo para tubería vertical Fuente: Cravino C, Annabella y Pérez B. 2011[5]

Flujo Burbuja. Los autores se basaron en el trabajo de Caetano (1985). Para el desarrollo del modelo se consideró el patrón de flujo burbuja dispersa separadamente al de flujo burbuja. Debido a la distribución uniforme de burbujas de gas en el líquido y la ausencia de deslizamiento entre las fases, el patrón burbuja dispersa se puede aproximar a un pseudomonofásico [6].

Ansari [5] considera la caída de presión por aceleración despreciable, ya que, según sus estudios, el flujo burbuja está dominado por una fase liquida incompresible por lo que no hay cambios significativos en la densidad de los fluidos [5] [6].

Flujo Tapón. En el caso de la componente de presión debida a la aceleración, según Ansari, la velocidad en la película se debe tomar en cuenta [5]. El líquido en 
el tapón se desacelera y su velocidad de ascenso lls $U$ disminuye hasta un valor igual a $l t b U$. El mismo líquido se acelera cuando sale de la película con una velocidad ltb $U$ hacia un tapón de líquido que se mueve a una velocidad $l l s U$. Si el cambio de velocidad sucede dentro del tapón entonces no ocurrirá una caída neta de presión en el mismo, mientras el flujo tapón sea estable. Es por esto que Ansari no toma en cuenta la componente de caída de presión debido a la aceleración [5].

Flujo Anular. Wallis presentó una ecuación para el cálculo del arrastre de líquido y el factor de fricción en función del espesor de película. Luego Hewitt y HallTaylor (1970) ofrecieron un análisis exhaustivo de los mecanismos involucrados en el flujo tipo anular. El valor de gradiente de presión total puede ser calculado por dos ecuaciones diferentes, presentadas por Alves (1988), ya que la caída de presión en la película de líquido debe ser la misma que la del núcleo [6].

\subsection{MODELO MECANÍSTICO DE XIAO ET AL. TUBERÍA HORIZONTAL}

Se presenta un modelo mecanístico unificado desarrollado para flujo gas-líquido en tuberías horizontales, el cual permite predecir el patrón de flujo presente en la tubería, características del patrón de flujo, la fracción de líquido o nivel y el gradiente de presión para los patrones de flujo estratificado, anular, intermitente y burbuja dispersa (Ver Figura 2)[7].

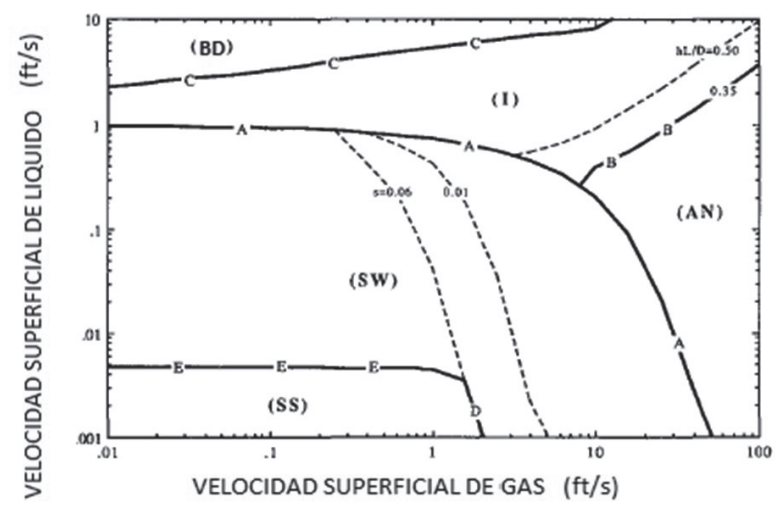

Figura 2. Mapa de patrones de flujo para tubería horizontal. Fuente: Xiao, J.J. 1990 [7].

Con respecto a lo anterior, el autor [7] expone que la existencia de los patrones de flujo:
Flujo Estratificado. Shoham (2000), explica que es el patrón de flujo donde el líquido fluye por el fondo y el gas por encima del líquido en la tubería, habiendo una separación de las dos fases debido al efecto causado por la fuerza de gravedad. El régimen estratificado se divide en: Estratificado liso y estratificado ondulado.

Estratificado liso (SS): La interfase líquido-gas tiene apariencia de una capa lisa con mínimas perturbaciones, de allí se observa la división entre ambas fases pero cuando se incrementa la velocidad del gas, las fuerzas interfaciales aumentan, formándose ondulaciones bruscas que originan una interfase ondulada y de allí deriva el término de Estratificado ondulado (SW).

Flujo intermitente. Se caracteriza por el flujo alterno de líquido y gas, cuando tapones de líquido ocupan por completo el área transversal de la tubería y están separados por burbujas de gas que viajan por la mitad superior del tubo y una película de líquido estratificado que fluye por el fondo de la misma.

Flujo Anular. De acuerdo a Shoham (2000), el flujo anular es aquel que surge a elevados velocidades del gas, se forma una parte central o núcleo de gas y una película de líquido alrededor de la periferia del tubo, la cual es más gruesa en la parte inferior del tubo. El arrastre de gotas de líquido por el gas es frecuente y presenta una subcategoría como es el régimen:

Anular ondulado: El cual es un proceso de transición en que la velocidad del gas y la mayor parte del líquido fluye como olas inestables en la parte inferior de la tubería, y la pared interior es mojable ya sea por el oleaje o por la disposición de las gotas.

Flujo Burbuja Dispersa. Shoham (2000), lo describe como el patrón de flujo en el cual existen pequeñas burbujas del gas dispersa en la fase líquida a lo largo de la tubería y se lleva a cabo a altos flujos de líquido con un amplio rango de velocidades del gas. Así mismo, bajo las condiciones de flujo burbuja dispersa, ambas fases se mueven con la misma velocidad, por lo que no ocurre deslizamiento entre estas. El flujo es considerado como homogéneo y tiene la característica de poseer la fracción volumétrica de líquido cercana a la unidad. 


\section{RESULTADOS}

Con el propósito de validar el desempeño global de la herramienta, se realizó una comparación de los resultados obtenidos por el software con el software PIPESIM, enfocado principalmente en el perfil de presión y de temperatura, para las correlaciones $\mathrm{y}$ modelos mecanísticos seleccionados para cada tipo de flujo.

A continuación se presentan los resultados obtenidos para flujo multifásico en tubería vertical:

Correlación empírica de Beggs \& Brill (1973) .Para esta correlación se utilizaron los siguientes datos de entrada:

Nota: Es importante resaltar que los datos de entrada de "source properties", "Properties flow line" y "Heat transfer" son los mismos para los diferentes modelos de tubería vertical, por esta razón no se presentan de nuevo estas imágenes en los modelos de Poettman \& Carpenter, y Ansari.

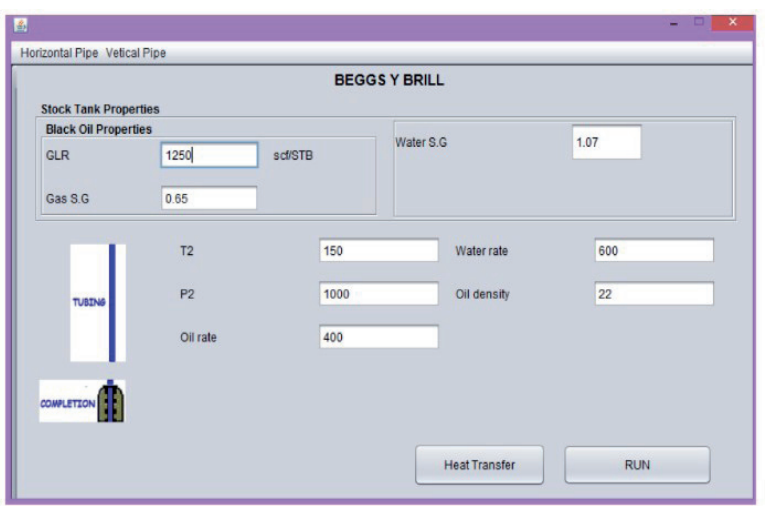

Figura 3. Datos de entrada correlación empírica Beggs \& Brill.

En la Tabla 5 se observan los resultados obtenidos para el perfil de presión y temperatura, con el respectivo error porcentual respecto al Software comercial PIPESIM.
Tabla 5. Datos de salida correlación empírica Beggs \& Brill

\begin{tabular}{|c|c|c|c|c|c|}
\hline \multicolumn{6}{|c|}{ CORRELACIÓN DE BEGGS \& BRILL } \\
\hline \multicolumn{2}{|c|}{ SOFTWARE } & \multicolumn{2}{|c|}{ PIPESIM } & \multirow{2}{*}{$\begin{array}{c}\text { Error } \\
(\%) \\
\text { presión }\end{array}$} & \multirow{2}{*}{$\begin{array}{l}\text { Error (\%) } \\
\text { temperatura }\end{array}$} \\
\hline $\begin{array}{c}\text { Presión } \\
\text { (psia) }\end{array}$ & $\begin{array}{l}\text { Temp. } \\
\left({ }^{\circ} \mathbf{F}\right)\end{array}$ & $\begin{array}{c}\text { Presión } \\
\text { (psia) }\end{array}$ & $\begin{array}{l}\text { Temp. } \\
\left({ }^{\circ} \mathbf{F}\right)\end{array}$ & & \\
\hline 514,7 & 77,5 & 623,1 & 80,0 & 21 & 3,2 \\
\hline 1214,7 & 81,0 & 1198,2 & 85,0 & 1,3 & 4,9 \\
\hline 1614,7 & 85,6 & 1676,3 & 88,8 & 3,8 & 3,8 \\
\hline 2014,7 & 90,1 & 2126,9 & 92,2 & 5,5 & 2,4 \\
\hline 2414,7 & 94,2 & 2510,4 & 95,1 & 3,9 & 0,9 \\
\hline 2614,7 & 96 & 2624,2 & 96 & 0,3 & 0 \\
\hline \multicolumn{4}{|c|}{ MEDIA } & 5,42 & 2,92 \\
\hline
\end{tabular}

Correlación empírica de Poettman y Carpenter. Para esta correlación se utilizaron los siguientes datos de entrada (Figura 4).

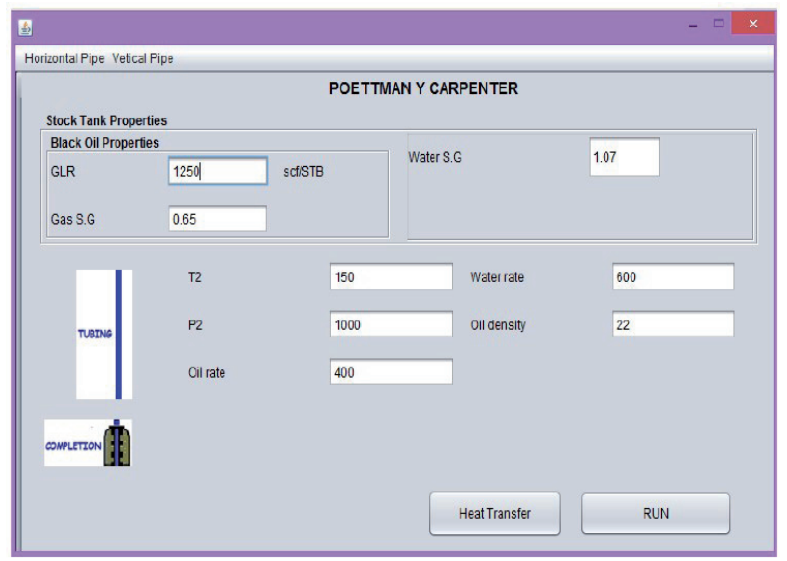

Figura 4. Datos de entrada correlación empírica Poettman y Carpenter.

En la Tabla 6 se pueden observar los resultados obtenidos para el perfil de presión y de temperatura, con el respectivo error porcentual respecto al software comercial PIPESIM.

Tabla 6. Datos de salida correlación empírica Poettman y Carpenter.

\begin{tabular}{|c|c|c|c|c|c|}
\hline \multicolumn{6}{|c|}{ CORRELACIÓN DE POETTMAN Y CARPENTER } \\
\hline \multicolumn{2}{|c|}{ SOFTWARE } & \multicolumn{2}{|c|}{ PIPESIM } & \multirow{2}{*}{$\begin{array}{c}\text { Error (\%) } \\
\text { presión }\end{array}$} & \multirow{2}{*}{$\begin{array}{c}\text { Error (\%) } \\
\text { temperatur: }\end{array}$} \\
\hline Presión (psia) & Temp $\left({ }^{\circ} \mathrm{F}\right)$ & Presión (psia) & Temp $\left({ }^{\circ} \mathrm{F}\right)$ & & \\
\hline 514,7 & 77,5 & 500,0 & 78,8 & 2,8 & 1,6 \\
\hline 1214,7 & 81,0 & 952,2 & 83,8 & 21,6 & 3,4 \\
\hline 1614,7 & 85,6 & 1334,9 & 87,8 & 17,3 & 2,6 \\
\hline 2014,7 & 90,1 & 1696,1 & 91,3 & 15,8 & 1,3 \\
\hline 2414,7 & 94,2 & 2046,5 & 94,5 & 15,2 & 0,2 \\
\hline 2614,7 & 96 & 2219,0 & 95 & 15,1 & 1,0 \\
\hline \multicolumn{4}{|c|}{ MEDIA } & 16,625 & 1,961 \\
\hline
\end{tabular}


Modelo Mecanístico de Ansari et al. (1994), Para esta correlación se utilizaron los siguientes datos de entrada (Figura 5).

En la Tabla 7 se pueden observar los resultados obtenidos para el perfil de presión y de temperatura, con el respectivo error porcentual respecto al software comercial PIPESIM.

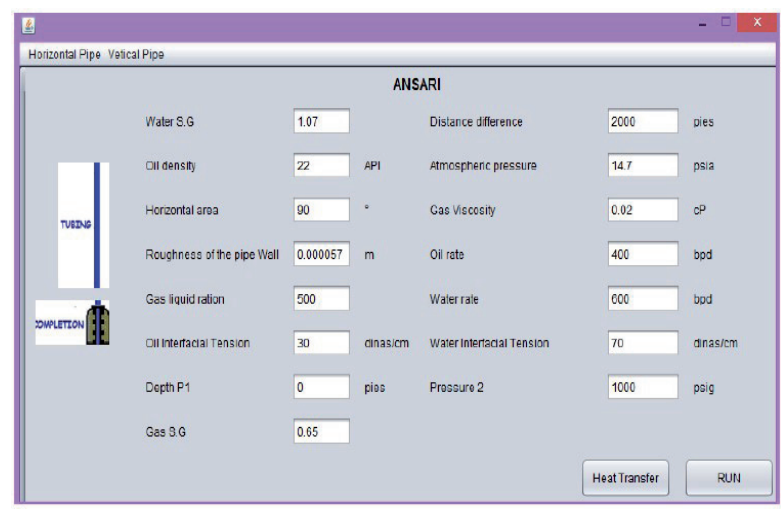

Figura 5. Datos de entrada Modelo Mecanístico de Ansari.

Tabla 7. Datos de salida modelo mecanístico de Ansari.

\begin{tabular}{|c|c|c|c|c|c|}
\hline \multicolumn{6}{|c|}{ MODELO DE ANSARI } \\
\hline \multicolumn{2}{|c|}{ SOFTWARE } & \multicolumn{2}{|c|}{ PIPESIM } & \multirow{2}{*}{$\begin{array}{c}\text { Error } \\
(\%) \\
\text { presión }\end{array}$} & \multirow{2}{*}{$\begin{array}{c}\text { Error (\%) } \\
\text { temperatura }\end{array}$} \\
\hline $\begin{array}{c}\text { Presión } \\
\text { (psia) }\end{array}$ & $\begin{array}{l}\text { Temp. } \\
\left({ }^{\circ} \mathbf{F}\right)\end{array}$ & $\begin{array}{c}\text { Presión } \\
\text { (psia) }\end{array}$ & $\begin{array}{l}\text { Temp } \\
\left({ }^{\circ} \mathbf{F}\right)\end{array}$ & & \\
\hline 414,4 & 77,5 & 516 & 79,1 & 24,5 & 1,9 \\
\hline 814,3 & 78,8 & 826,5 & 83,1 & 1,4 & 5,3 \\
\hline 1214,3 & 83,2 & 1160,1 & 86,7 & 4,4 & 4,1 \\
\hline 1614,3 & 87,8 & 1494,8 & 89,9 & 7,4 & 2,3 \\
\hline 2014,3 & 92,2 & 1827,4 & 92,9 & 9,2 & 0,7 \\
\hline 2414,3 & 96 & 2135 & 95,6 & 11,5 & 0,4 \\
\hline 2814,28 & 99,5 & 2598,4 & 98,4 & 7,6 & 1,1 \\
\hline \multicolumn{4}{|c|}{ MEDIA } & 9,1135 & 2,5368 \\
\hline
\end{tabular}

A continuación, se presentan los resultados obtenidos para flujo multifásico en tubería horizontal:
Correlación empírica de Eaton et al. (1967). Para esta correlación se utilizaron los siguientes datos de entrada (Figura 6,8,). En la tabla 8 y 9 se pueden observar los datos de salida. Nota: Los valores de entrada para "Source properties", "properties flowline", "Heat transfer" tubería no aislada y tubería aislada, son los mismos para los demás modelos de tubería horizontal, por esta razón no se repiten estas imágenes en estos (Beggs \& Brill y Xiao).

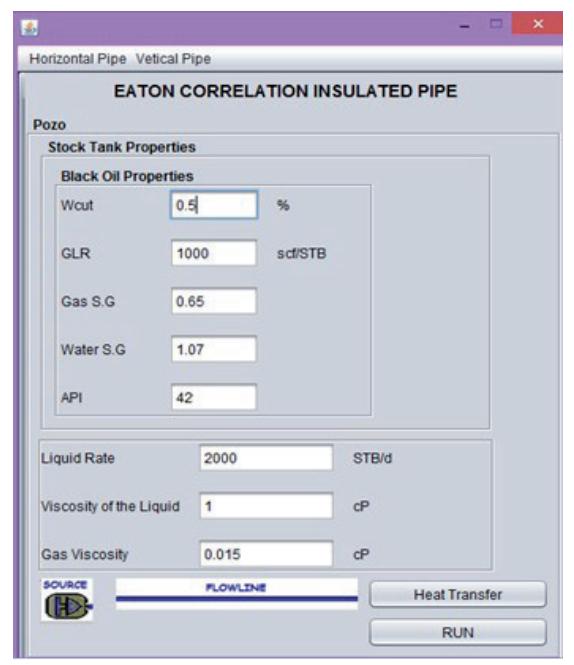

Figura 6. Datos de entrada correlación de Eaton.

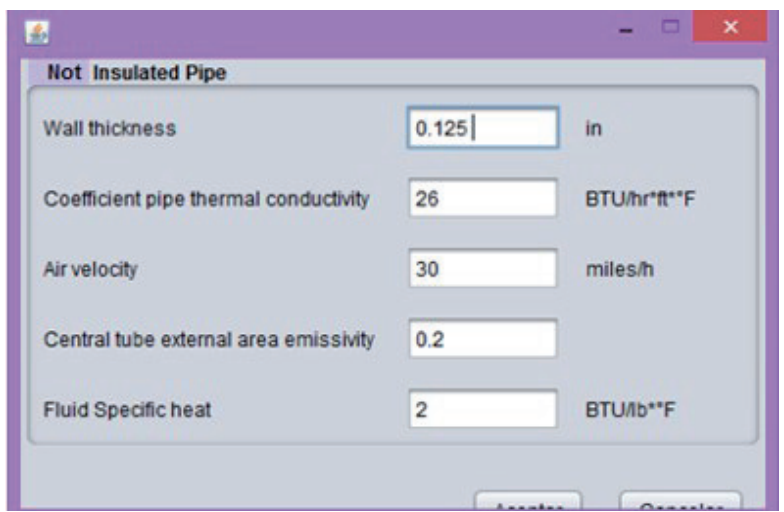

Figura 7. Datos de entrada correlación de Eaton transferencia de calor tubería no aislada.

Tabla 8. Datos de salida correlación Eaton tubería no aislada.

\begin{tabular}{|cccccc|}
\hline \multicolumn{7}{c}{ SOFTWARE } & \multicolumn{2}{c|}{ PIPESIM } & Error (\%) presión & Error (\%) temperatura \\
\hline Presión (psia) & Temp. $\left({ }^{\circ} \mathbf{F}\right)$ & Presión (psia) & Temp. $\left({ }^{\circ} \mathbf{F}\right)$ & 0 & 1,3 \\
\hline 850 & 118,3 & 850 & 119,9 & 0,18 & 18,9 \\
847,6 & 109,4 & 846,0 & 88,7 & 0,34 & 34,7 \\
845,3 & 101,9 & 842,3 & 66,5 & 0,40 & 34,7 \\
843,7 & 97,5 & 840,3 & 63,6 & 0,42 & 34,8 \\
842,8 & 95,5 & 839,2 & 62,2 & 0,44 & 34,9 \\
\hline 841,9 & 93,6 & 838,2 & 60,9 & 0,26 & 25,04 \\
\hline
\end{tabular}


Tabla 9. Datos de salida correlación Eaton tubería aislada.

\begin{tabular}{|c|c|c|c|c|c|}
\hline \multicolumn{6}{|c|}{ CORRELACIÓN DE EATON PARA TUBERÍA AISLADA } \\
\hline \multicolumn{2}{|c|}{ SOFTWARE } & \multicolumn{2}{|c|}{ PIPESIM } & \multirow{2}{*}{$\begin{array}{c}\text { Error (\%) } \\
\text { presión }\end{array}$} & \multirow{2}{*}{$\begin{array}{c}\text { Error (\%) } \\
\text { temperatura }\end{array}$} \\
\hline Presión (psia) & Temp. $\left({ }^{\circ} \mathbf{F}\right)$ & Presión (psia) & Temp. $\left({ }^{\circ} \mathbf{F}\right)$ & & \\
\hline 850,0 & 119,8 & 850 & 119,9 & 0,0 & 0,11 \\
\hline 847,7 & 119,0 & 846,3 & 119,2 & 0,17 & 0,19 \\
\hline 846,9 & 118,7 & 845,0 & 119 & 0,22 & 0,21 \\
\hline 845,4 & 118,2 & 842,9 & 118,5 & 0,29 & 0,27 \\
\hline 843,8 & 117,6 & 841,0 & 118,0 & 0,32 & 0,35 \\
\hline 842,0 & 117,1 & 839,2 & 117,6 & 0,33 & 0,40 \\
\hline \multicolumn{4}{|c|}{ MEDIA } & 0,223 & 0,256 \\
\hline
\end{tabular}

Correlación empírica de Beggs \& Brill (1973). Para esta correlación se utilizaron los siguientes datos de entrada (Figura 9).

En la Tabla 10 y 11 se pueden observar los resultados obtenidos para el perfil de presión y de temperatura, con el respectivo error porcentual respecto al software comercial PIPESIM.

Modelo mecanístico de Xiao et al (1990). Para la validación del modelo mecanístico de Xiao en tuberías horizontales, se utilizaron los siguientes datos de entrada (Figura 10).

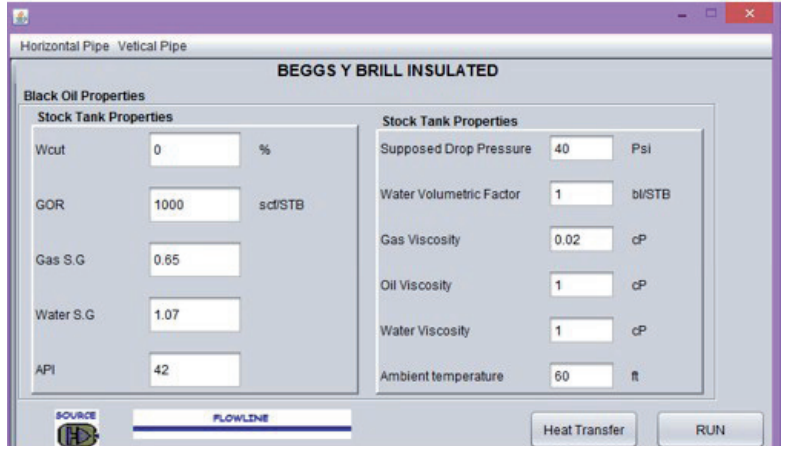

Figura 8. Datos de entrada correlación Beggs \& Brill (flujo tubería Horizontal).

Tabla 10. Datos de salida correlación Beggs \& Brill tubería No aislada.

\begin{tabular}{|c|c|c|c|c|c|}
\hline \multicolumn{6}{|c|}{ CORRELACIÓN DE BEGGS \& BRILL PARA TUBERÍA NO AISLADA } \\
\hline \multicolumn{2}{|c|}{ SOFTWARE } & \multicolumn{2}{|c|}{ PIPESIM } & \multirow{2}{*}{$\begin{array}{c}\text { Error (\%) } \\
\text { presión }\end{array}$} & \multirow{2}{*}{ Error $(\%)$ temperatura } \\
\hline Presión (psia) & Temp. $\left({ }^{\circ} \mathbf{F}\right)$ & Presión (psia) & Temp. $\left({ }^{\circ} \mathbf{F}\right)$ & & \\
\hline 850 & 118,3 & 850 & 119,9 & 0,0 & 1,3 \\
\hline 847,6 & 109,4 & 846,2 & 88,6 & 0,16 & 19,0 \\
\hline 846,8 & 106,8 & 844,9 & 78,0 & 0,21 & 26,9 \\
\hline 845,3 & 101,9 & 843,1 & 66,3 & 0,26 & 34,8 \\
\hline 843,7 & 97,5 & 841,8 & 63,6 & 0,23 & 34,8 \\
\hline 842,8 & 95,5 & 841,1 & 62,2 & 0,19 & 34,9 \\
\hline \multicolumn{4}{|c|}{ MEDIA } & 0,179 & 25,09 \\
\hline
\end{tabular}

Tabla 11. Datos de salida correlación Beggs \& Brill tubería aislada.

\begin{tabular}{|cccccc|}
\hline \multicolumn{5}{|c}{ CORRELACIÓN DE BEGGS \& BRILL PARA TUBERÍA AISLADA } \\
\hline \multicolumn{5}{|c}{ SOFTWARE } & \multicolumn{2}{c|}{ PIPESIM } & $\begin{array}{c}\text { Error (\%) } \\
\text { presión }\end{array}$ & $\begin{array}{c}\text { Error (\%) } \\
\text { temperatura }\end{array}$ \\
\hline Presión (psia) & Temp. $\left({ }^{\circ} \mathbf{F}\right)$ & Presión (psia) & Temp. $\left({ }^{\circ} \mathbf{F}\right)$ & & 0,11 \\
\hline 850,0 & 119,8 & 850,0 & 119,9 & 0,0 & 0,18 \\
\hline 847,7 & 119,0 & 846,5 & 119,2 & 0,14 & 0,23 \\
\hline 846,2 & 118,4 & 844,2 & 118,7 & 0,24 & 0,26 \\
\hline 845,4 & 118,2 & 843,7 & 118,5 & 0,20 & 0,33 \\
\hline 843,8 & 117,6 & 842,7 & 118,0 & 0,12 & 0,36 \\
\hline 842,9 & 117,3 & 842,2 & 117,8 & 0,08 & $\mathbf{0 , 2 4 8}$ \\
\hline
\end{tabular}




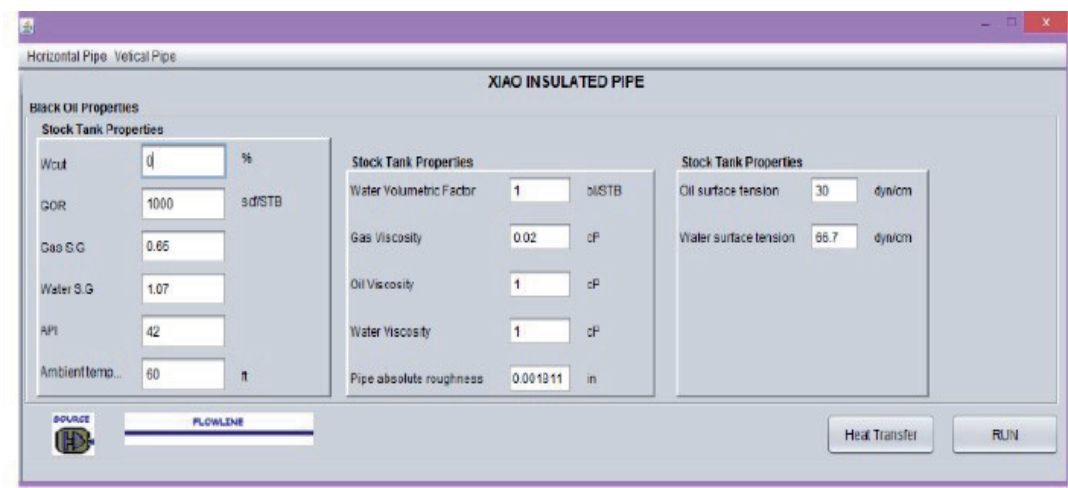

Figura 9. Datos de entrada modelo mecanistico de Xiao.

Tabla 12. Datos de salida Modelo mecanístico de Xiao tubería no aislada.

\begin{tabular}{|c|c|c|c|c|c|}
\hline \multicolumn{6}{|c|}{ MODELO MECANÍSTICO DE XIAO PARA TUBERÍA NO AISLADA } \\
\hline \multicolumn{2}{|c|}{ SOFTWARE } & \multicolumn{2}{|c|}{ PIPESIM } & \multirow{2}{*}{$\begin{array}{c}\text { Error (\%) } \\
\text { presión }\end{array}$} & \multirow{2}{*}{$\begin{array}{c}\text { Error (\%) } \\
\text { temperatura }\end{array}$} \\
\hline Presión (psia) & Temp. $\left({ }^{\circ} \mathbf{F}\right)$ & Presión (psia) & Temp $\left({ }^{\circ} \mathbf{F}\right)$ & & \\
\hline 850,0 & 118,3 & 850,0 & 119,9 & 0,00 & 1,37 \\
\hline 839,6 & 104,3 & 844,0 & 67,7 & 0,52 & 35,04 \\
\hline 835,1 & 99,6 & 842,1 & 65,7 & 0,83 & 34,09 \\
\hline 832,8 & 97,5 & 841,0 & 64,8 & 0,97 & 33,52 \\
\hline 830,5 & 95,5 & 840,3 & 63,2 & 1,18 & 33,85 \\
\hline 828,1 & 93,6 & 839,7 & 61,3 & 1,39 & 34,53 \\
\hline \multicolumn{4}{|c|}{ MEDIA } & 0,597 & 24,85 \\
\hline
\end{tabular}

Tabla 13. Datos de salida Modelo mecanístico de Xiao tubería aislada.

\begin{tabular}{|cccccc|}
\hline \multicolumn{7}{|c|}{ MODELO MECANÍSTICO DE XIAO PARA TUBERÍA AISLADA } \\
\hline \multicolumn{2}{|c|}{ SOFTWARE } & \multicolumn{2}{c|}{ PIPESIM } & $\begin{array}{c}\text { Error (\%) } \\
\text { presión }\end{array}$ & $\begin{array}{c}\text { Error (\%) } \\
\text { temperatura }\end{array}$ \\
\hline Presión (psia) & Temp. $\left({ }^{\circ} \mathbf{F}\right)$ & Presión (psia) & Temp. $\left({ }^{\circ} \mathbf{F}\right)$ & 0,00 & 0,11 \\
\hline 850 & 119,8 & 850 & 119,9 & 0,12 & 0,07 \\
845,9 & 119,3 & 846,9 & 119,3 & 0,25 & 0,12 \\
841,8 & 118,7 & 843,9 & 118,8 & 0,17 \\
837,5 & 118,2 & 841,3 & 118,4 & 0,44 & 0,27 \\
828,8 & 117,1 & 837,7 & 117,4 & 1,07 & 0,150 \\
\hline
\end{tabular}

\section{DISCUSIÓN}

\section{TUBERÍA VERTICAL}

Correlación empírica de Beggs \& Brill. Se obtuvieron los datos de presión y temperatura, y a partir de estos valores se determinó el error porcentual tanto para la presión como para la temperatura tomando como valores reales los arrojados por PIPESIM y como valores experimentales los arrojados por el software, dando márgenes de error medio aceptables de 2,92\% para el perfil de temperatura y $5,42 \%$ para el perfil de presión (Figura 11, 12).

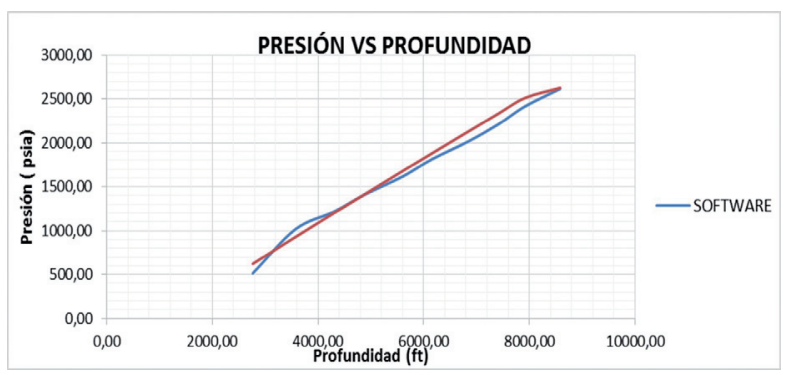

Figura 10. Perfil de presión correlación Beggs \& Brill 


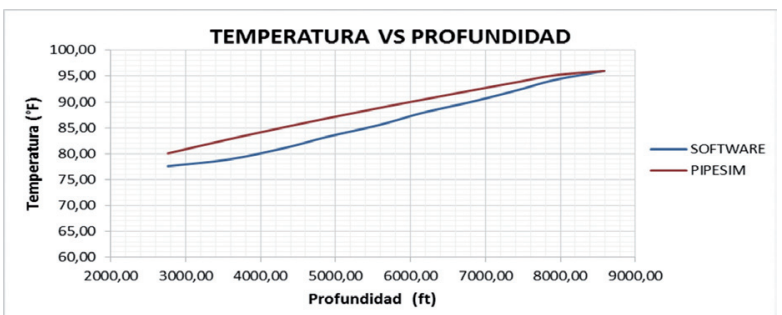

Figura 11. Perfil de Temperatura correlación Beggs \& Brill

Correlación empírica de Poettman y Carpenter. Se obtuvieron márgenes de error medio aceptables de $1,96 \%$ para el perfil de temperatura y $16,62 \%$ para el perfil de presión (Figura 13, 14).

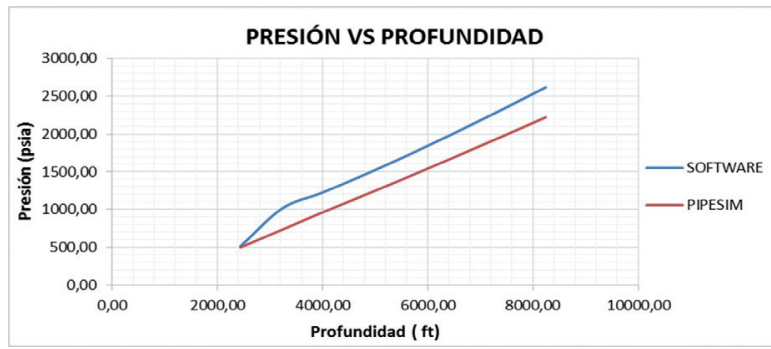

Figura 12. Perfil de Presión correlación Poettman y Carpenter.

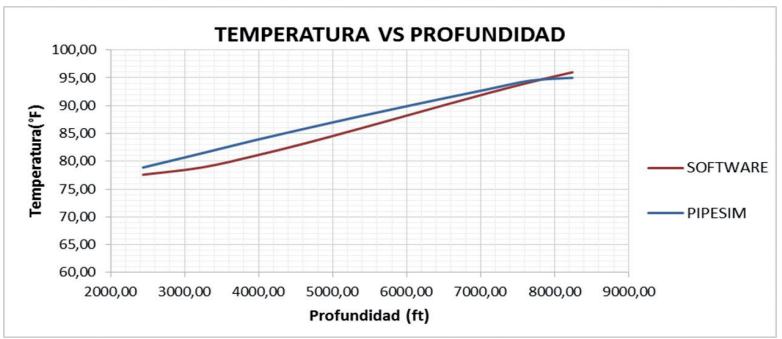

Figura 13. Perfil de Temperatura correlación Poettman y Carpenter.

Modelo mecanístico de Ansari. Se obtuvieron márgenes de error medianamente aceptables de $2,54 \%$ para el perfil de temperatura y $9,11 \%$ para el perfil de presión (Figura 15, 16).

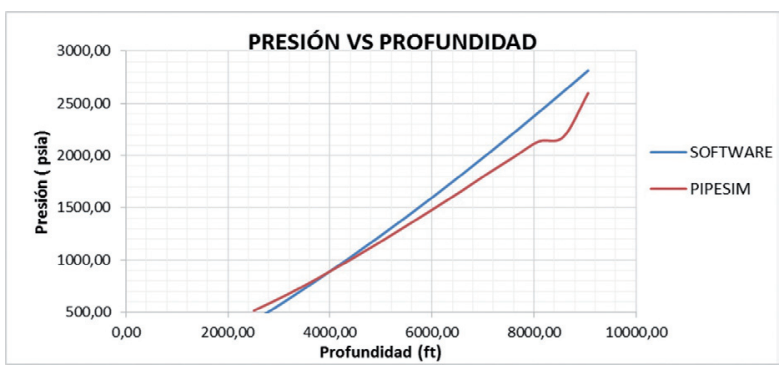

Figura 14. Perfil de Presión modelo mecanístico de Ansari.

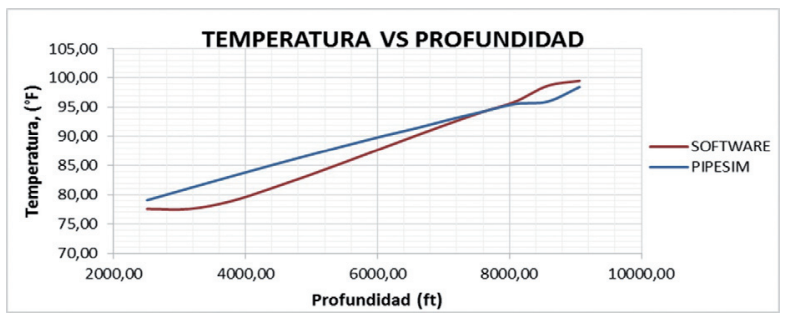

Figura 15. Perfil de Temperatura modelo mecanístico de Ansari.

\section{TUBERÍA HORIZONTAL}

Correlación empírica de Eaton. Para el caso de tubería no aislada y de tubería aislada, se obtuvieron los datos de presión y temperatura, a partir de estos valores se determinó el error porcentual tanto para la presión como para la temperatura tomando como valores reales los arrojados por PIPESIM y como valores experimentales los arrojados por el software, dando márgenes de error máximo de $25,0423 \%$ para el perfil de temperatura y un máximo de $0,2681 \%$ para el perfil de presión.

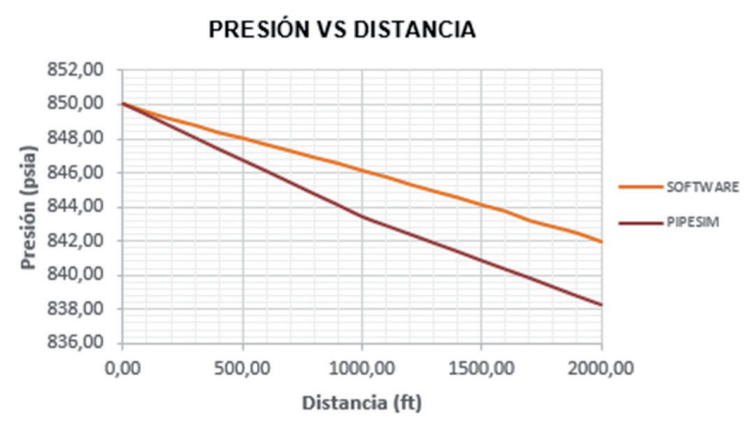

Figura 16. Perfil de presión para tubería No aislada correlación de Eaton.

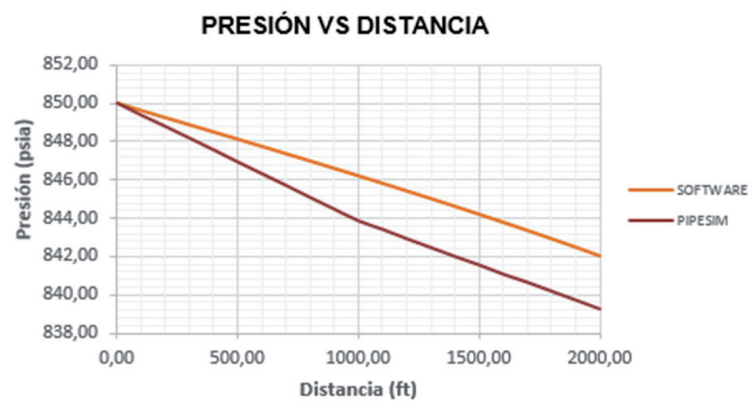

Figura 17. Perfil de Presión para tubería aislada correlación de Eaton. 


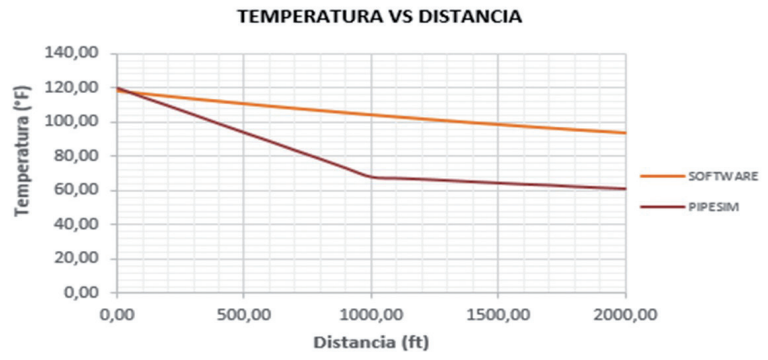

Figura 18. Perfil de Temperatura para tubería No aislada correlación de Eaton.

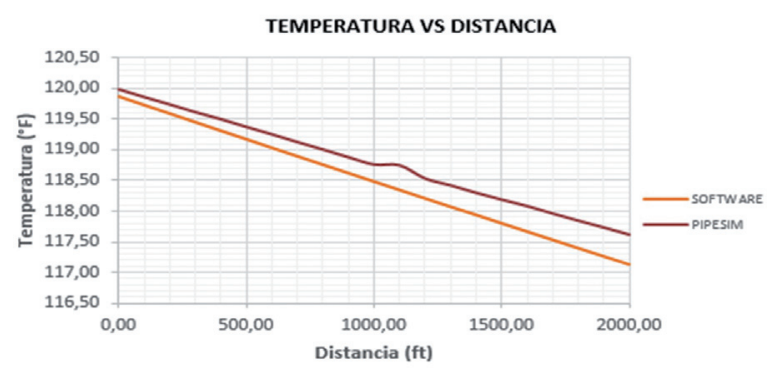

Figura 19. Perfil de Temperatura para tubería aislada correlación de Eaton.

Correlación empírica de Beggs \& Brill. Tanto para tubería no aislada como tubería aislada, se obtuvieron los datos de presión y temperatura, a partir de estos valores se determinó el error porcentual para la presión y la temperatura tomando como valores reales los proporcionados por PIPESIM y como valores experimentales los proporcionados por el software, brindando márgenes de error máximo de $25,0956 \%$ para el perfil de temperatura y un máximo de $0,1792 \%$ para el perfil de presión.

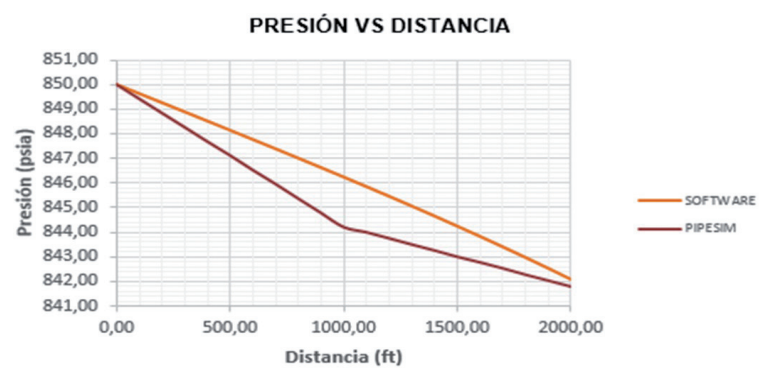

Figura 20. Perfil de presión para tubería No aislada correlación de Beggs \& Brill- Tubería horizontal

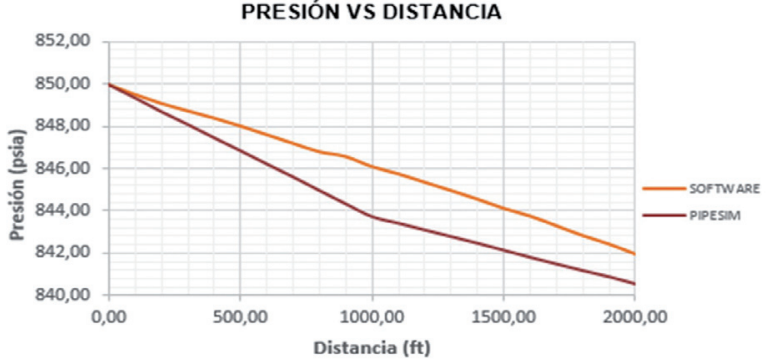

Figura 21. Perfil de presión para tubería aislada correlación de Beggs \& Brill- Tubería horizontal

Modelo Mecanístico de Xiao. Para tubería no aislada y tubería aislada, se obtuvieron los datos de presión y temperatura, a partir de estos valores se halló el error porcentual tanto para la presión como para la temperatura tomando como valores reales los arrojados por PIPESIM y como valores experimentales los arrojados por el software, dando perfiles de error máximo de $24,8537 \%$ para el perfil de temperatura y un máximo de $0,5979 \%$ para el perfil de presión.

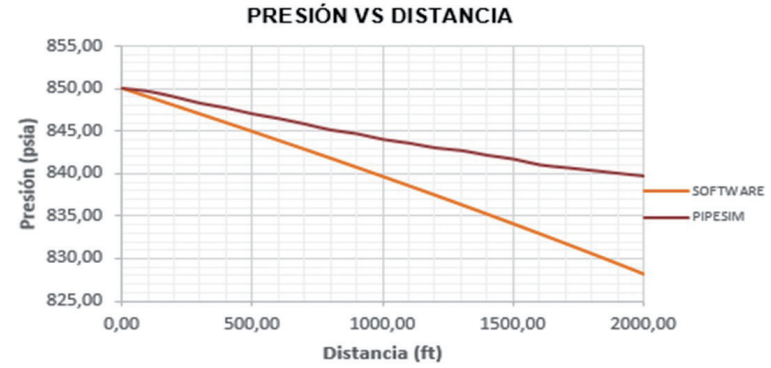

Figura 22. Perfil de presión para tubería No aislada Modelo mecanístico de Xiao.

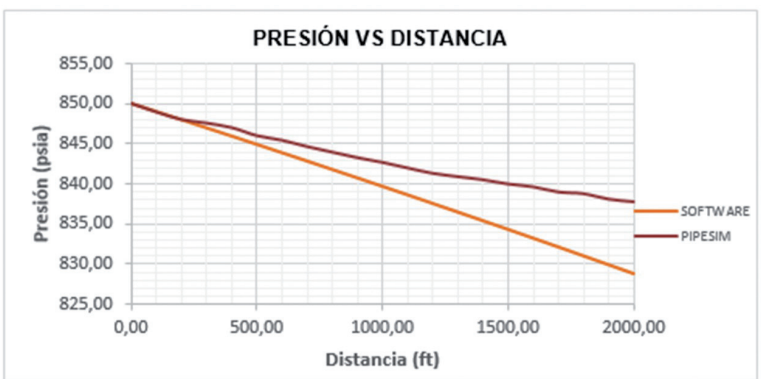

Figura 23. Perfil de presión para tubería aislada Modelo mecanístico de Xiao- Tubería horizontal 


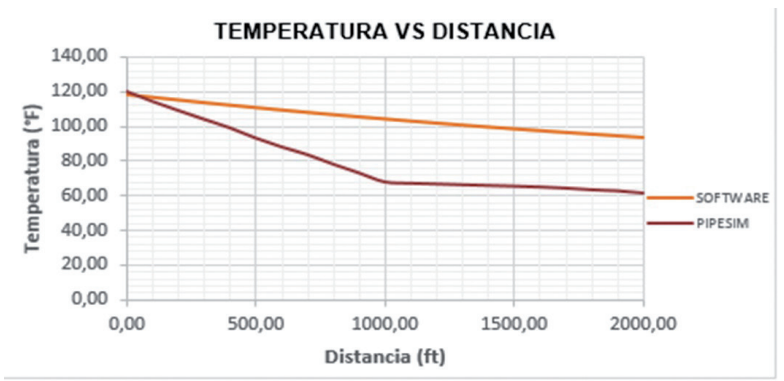

Figura 24. Perfil de temperatura para tubería No aislada Modelo mecanístico de Xiao.

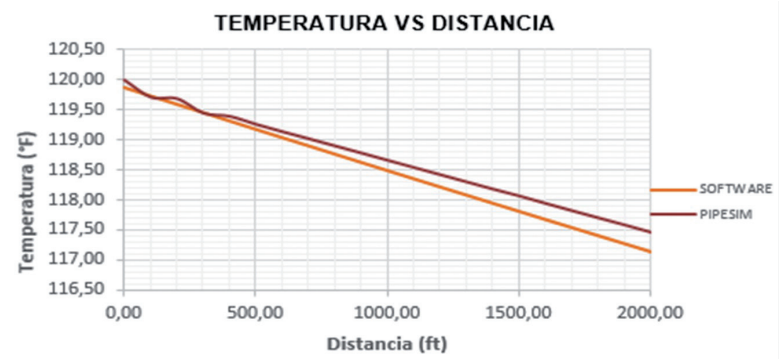

Figura 25. Perfil de Temperatura para tubería aislada Modelo mecanístico de Xiao.

La similitud con los datos reales, podría mejorarse si se evalúan las diferentes correlaciones y modelos que presenta la herramienta, para seleccionar el más ajustable a las condiciones del pozo ya que no todos presentan el mismo comportamiento, por lo cual se sugiere hacer un análisis de las propiedades, para saber en cuales rangos de aplicabilidad de las diferentes correlaciones puede ser sometido cada pozo.

\section{CONCLUSIONES}

El Software para el modelamiento del flujo multifásico en tubería vertical y horizontal desarrollado, consta de algunos de los modelos más aplicados a nivel académico, desarrollado además, con una interfaz gráfica amigable con el usuario, que facilita su uso.

Se llevó a cabo la validación de los resultados de perfil de presión y de temperatura, obtenidos por el software, con el software comercial PIPESIM, comparando tales resultados, arrojando márgenes de error aceptables en la mayoría de los casos.

Entre los modelos para flujo en tubería vertical, respecto a la predicción de presión, la correlación de Beggs y Brill presenta mayor similitud con los resultados de PIPESIM, mientras que la correlación de Poettman y Carpenter obtuvo el porcentaje de error más alto, debido a que este último es más extenso y emplea gráficas complejas en la programación.
Para tubería vertical en la predicción de temperatura, la correlación de Poettman y Carpenter obtuvo menor error mientras que en Beggs y Brill se obtuvo el mayor, es importante resaltar que en los 2 modelos y la correlación se obtuvieron valores muy cercanos al software comercial.

En los modelos para flujo en tubería horizontal respecto a la predicción de presión, la correlación empírica de Beggs y Brill presenta mayor aproximación con los resultados proporcionados por PIPESIM, mientras que el modelo mecanístico de Xiao presentó márgenes de error más altos, sin embargo todos los errores calculados están alrededor del 1\%, es decir los resultados tiene una alta confiabilidad.

Para tubería horizontal en la predicción de temperatura, la correlación de Beggs y Brill obtuvo el mayor error porcentual, mientras que en el modelo Mecanístico de Xiao se obtuvo el error porcentual, sin embargo se puede apreciar que los errores son altos alrededor de $25 \%$, esto se debe a que en las correlaciones del software desarrollado, se emplea menos parámetros que en PIPESIM, lo cual a la vez facilita al usuario ingresar valores de entrada, ya que no siempre se cuenta con toda la información.

Éste proyecto logró el desarrollo de la primera versión, alcanzando los objetivos planteados. Además, con el desarrollo de ésta aplicación la escuela de Ingeniería de Petróleos tiene un modelo sobre el cual trabajar para agregar nuevas funcionalidades.

\section{REFERENCIAS}

1. BEDOYA R., Sandra V., Fontecha C., Diana C. Desarrollo de una herramienta computacional con correlaciones de flujo multifásico aplicando el factor de ajuste para la viscosidad de crudos pesados. Trabajo de grado. Bucaramanga. Universidad Industrial de Santander. Facultad de ingenierías fisicoquimicas. Escuela de Ingenieria de Petróleos, 2010. Paginas 21-22.

2. TORRES C., Juan M., TRAUWVITZ R., Edgar F. Flujo multifásico en tuberías. Trabajo de grado. México D.F. Universidad Nacional Autonoma de México. Facultad de ingeniería. División de ciencias de la Tierra, 2008. Página 122.

3. ARANGO M. Johana, GONZALES F., Katlyn L. Predicción de pérdidas de presión y perfiles de temperatura en pozos geotérmicos usando modelos 
mecanísticos. Trabajo de grado. Bucaramanga. Universidad Industrial de Santander. Facultad de Ingenierias Fisicoquimicas. Escuela de Ingeniería de Petróleos, 2011. Página 68.

4. ROMERO A., Alejandro J., SALAZAR P., Dimas B. Herramienta computacional para el estudio del comportamiento o del flujo multifásico con transferencia de calor en líneas de flujo. Trabajo de grado. Caracas, 2007. Página 13.

5. ANSARI, A.M. A Comprehensive Mechanistic Model for upward Two-phase Flow in Wellbores. Paper SPE 20630, September 1990.

6. CRAVINO C, Annabella y Pérez B, AID. Evaluación de modelos homogéneos, de correlación y mecanicistas en la predicción de la caída de presión de flujo bifásico de gas y de líquido en tuberías verticales. Trabajo de grado. Caracas, 2007.

7. XIAO, J. J. A Comprehensive Mechanistic Model for Two-phase Flow in Pipelines, Paper SPE 20631, September 1990.

8. GUILARTE P., María G., LISCANO C., VICEYNI. Determinación de gradientes de presión para el estudio del comportamiento de una línea de flujo multifásico mediante el empleo de correlaciones en tuberías horizontales e inclinadas. Trabajo de grado. Barcelona. Universidad de Oriente, Núcleo de Anzuategui. Escuela de Ingeniería y Ciencias Aplicadas. Departamento de Ingeniería Quimica, 2011.

9. Brill, J. P., MUKHERJEE H. Multiphase flow in Wells, Richardson, Texas, 1999.

10. SCHLUMBERGER Information Solution, PIPESIM Fundamentals, Workflow/Solutions Training, Houston, Texas, February, 2012.

11. CABARCAS S. Manuel. Transferencia de calor: coeficiente global de transferencia de calor para pozos, fundamentación teórica, Universidad Industrial de Santander, 2010.

12. YAHAYA, Anes Usman, ABOALLAH Algahtani. Comparative study between empiriccorrelation \& mechanistic model of vertical multiphase flow, paper SPE 136931, 2010.

13. RUIZ R., BRITO A., MARQUEZ J. Evaluation of multiphase flow models to predict pressure gradient in vertical pipes with highly viscous liquids, paper SPE 169328, May 2014.

14. GARCIAF; SEGURA, J.; MORAN, R. Transferencia de calor en flujo bifásico gas-liquido parte I: Pozos y tuberías horizontales, revista de la facultad de Ingenieria de la U.C.V., Vol. 19, $\mathrm{N}^{\circ}$ 3, 2004. 

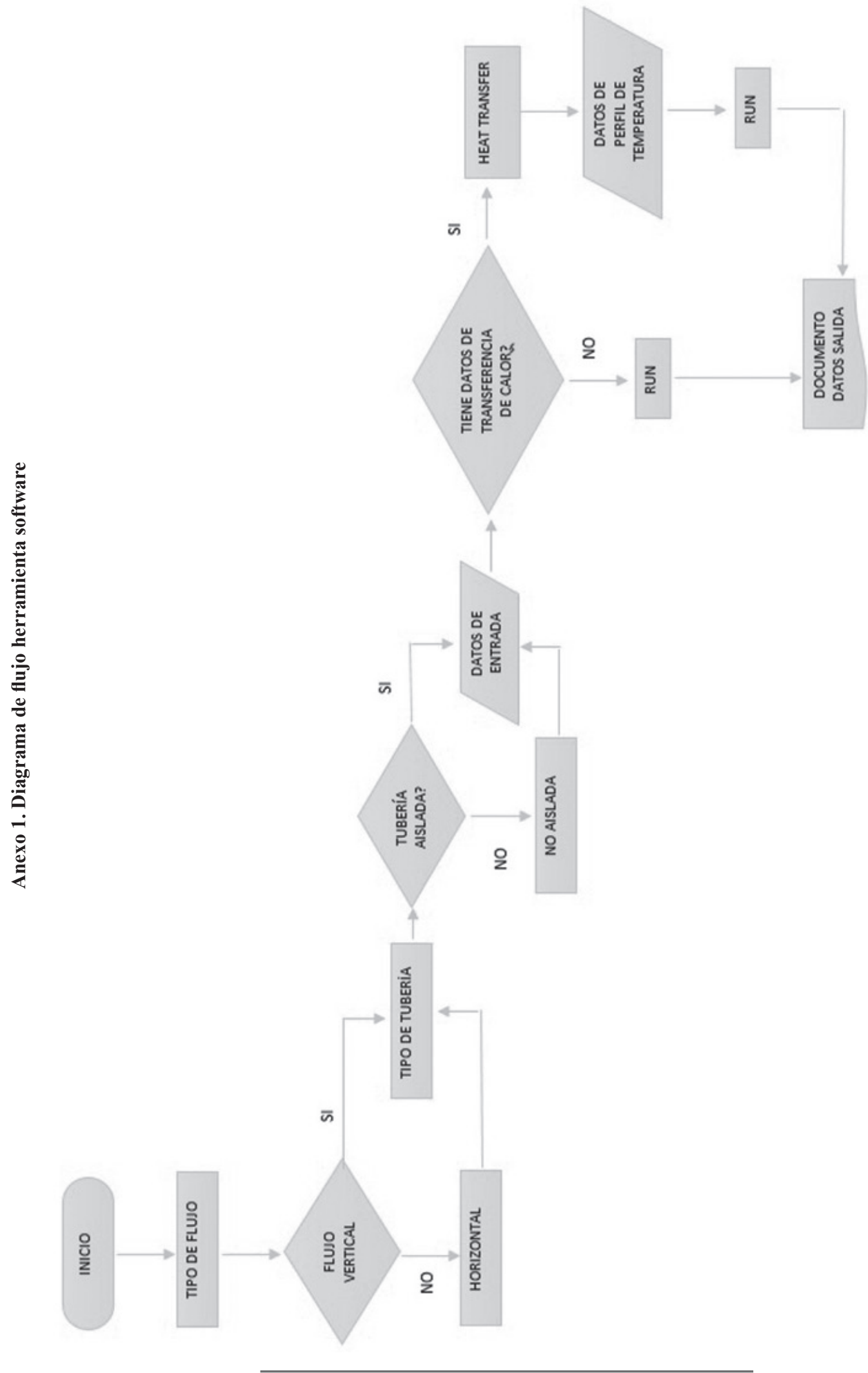

Recepción: 15 de enero de 2016

Aceptación: 18 de marzo de 2016 